nachgewiesen sein musste. Die Patienten erhielten entweder $75 \mathrm{mg}$ Clopidogrel oder Placebo. Alle Patienten wurden zusätzlich mit $325 \mathrm{mg}$ ASS behandelt. Der primäre Endpunkt war jeder erneute Schlaganfall unabhängig davon ob ischämischer Schlaganfall oder intrakranielle Blutung. 1.503 Patienten erhielten die ASS-Monotherapie und 1.517 die Kombinationstherapie. Den Endpunkt „alle Schlaganfälle" erreichten unter ASS-Monotherapie 138 und unter Kombinationstherapie 125 Patienten. Dies entspricht jährlichen Raten von 2,7\% und 2,5\%. Dieser Unterschied war statistisch nicht signifikant. Es ergaben sich auch keine Unterschiede, wenn ischämische Insulte und intrakranielle Blutungen getrennt ausgewertet wurden. Auch bei Herzinfarkten und vaskulären Todesfällen ergaben sich keine Unterschiede. Schwerwiegende Blutungskomplikationen traten bei 56 Patienten in der ASS-Monotherapie und bei 105 in der Kombinationstherapie auf. Dies war statistisch signifikant. Es traten 77 Todesfälle unter ASS-Monotherapie und 113 unter Kombinationstherapie auf. Dies war ebenfalls statistisch signifikant.

Kommentar: Diese Studie bestätigt die Ergebnisse der bereits vor einigen Jahren durchgeführten Studien MATCH und CHARISMA. In der MATCH-Studie wurden ASS plus Clopidogrel mit Clopidogrel und in der CHARISMA-Studie wurden ASS plus Clopidogrel mit ASS verglichen. Allen Studien gemeinsam ist, dass die Kombinationstherapie einer Monotherapie nicht überlegen (und nicht unterlegen) ist, aber zu vermehrten Blutungskomplikationen führt. Dies ist ein bemerkenswerter Unterschied zur kardiologischen Indikation. Für das akute Koronarsyndrom ist nämlich die Langzeitprophylaxe von ASS und Clopidogrel einer Monotherapie mit ASS eindeutig überlegen. Es muss allerdings berücksichtigt werden, dass die ASS-Dosis in dieser Studie mit $325 \mathrm{mg}$ sehr hoch war. Dennoch gilt weiterhin, dass in der Langzeittherapie nach einem Schlaganfall jedweder Art nur ASS, Clopidogrel oder die Kombination von ASS und Dipyridamol sinnvollerweise in Betracht kommen. Professor Stefan Evers

Benavente OR et al. (SPS3 Investigators): Effects of clopidogrel added to aspirin in patients with recent lacunar stroke. N Engl J Med 2012; 367: $817-825$

\title{
Magnesium ist wirkungslos bei der akuten Subarachnoidalblutung
}

\section{Nach dieser Studie scheint es nun endgültig festzustehen, dass Magnesium bei der akuten SAB keinen Nutzen hat.}

$D^{i}$ e Sterblichkeit einer Subarachnoidalblutung (SAB) innerhalb eines Monats beträgt immer noch bis zu $44 \%$. Die wichtigste Komplikation einer SAB ist die zerebrale Ischämie durch Vasospasmen. Die einzige bisher zugelassene wirksame Therapie zur Prävention von Vasospasmen ist die intravenöse Gabe von Nimodipin. Immer wieder ist auch Magnesium diskutiert worden, das im Tierexperiment in der Lage ist, Vasospasmen bei einer $\mathrm{SAB}$ zu reduzieren. Daher ist in dieser randomisierten, placebokontrollierten Phase-III-Studie untersucht worden, ob Magnesium die Prognose nach einer SAB verbessert. Patienten mit einer SAB wurden entweder mit $64 \mathrm{mmol}$ intravenösem Magnesium pro Tag oder mit Placebo über 20 Tage behandelt. Der primäre Endpunkt war definiert als ein Wert von 4 oder 5 auf der modifizierten Rankin-Skala nach 90 Tagen oder jedweder Tod. 1.204 Patienten wurden eingeschlossen; $606 \mathrm{~Pa}$ tienten erhielten Magnesium und 597 Placebo. Den primären Endpunkt erreichten 158 Patienten in der Magnesiumgruppe $(26,2 \%)$ verglichen mit 151 in der Placebogruppe (25,3\%). Dieser Unterschied war statistisch nicht signifikant. Auch eine Metaanalyse von sieben ähnlichen Studien mit insgesamt 2.047 Patienten zeigte keinen Nutzen von Magnesium im Vergleich zu Placebo in Bezug auf die Prognose nach einer SAB.

Kommentar: Leider ist diese bislang größte Studie zu Magnesium negativ verlaufen, sodass man nun wohl definitiv sagen kann, dass Magnesium bei der akuten SAB keinen Nutzen hat. Magnesium ist zwar im Tierexperiment wirksam und hatte in kleineren Phase-II-Studien einen positiven Trend bei Patienten mit SAB gezeigt. Wie so häufig bei Schlaganfallstudien konnten allerdings die Ergebnisse aus den Phase-II-Studien nicht in Phase-III-Studien reproduziert werden. Einzige sinnvolle Option bleibt also weiterhin die Gabe von intravenösem Nimodipin.

Professor Stefan Evers

Dorhout Mees SM et al.: Magnesium for aneurysmal subarachnoid haemorrhage (MASH-2): a randomised placebo-controlled trial. Lancet 2012; 380: 44-49 